\title{
Zonificación ambiental como marco de referencia para el reordenamiento del territorio: caso aplicado al municipio Uribante (Táchira, Venezuela)
}

\author{
Environmental zoning as a reference framework \\ to the territorial reshaping: Case Uribante \\ municipality (Táchira, Venezuela)
}

Damary Elizabeth Chacón Casique*

\section{Resumen}

Muestra parte de los resultados obtenidos en la investigación desarrollada por la autora para optar al título de Magíster en Geografía con énfasis en Ordenamiento Territorial. La investigación responde a la necesidad latente de definir una estrategia orientada a identificar y analizar la problemática ambiental de un área determinada del territorio venezolano: el municipio Uribante, estado Táchira. Se pretende, de alguna manera, contribuir con una propuesta de zonificación ambiental aplicada a esta área en particu lar, que permita definir y proponer alternativas para su desarrollo, que sean coherentes con los principios de sostenibilidad.

\footnotetext{
* Licenciada en Educación, mención Geografía y Ciencias de la Tierra, Universidad de los Andes (Táchira, Venezuela). Magíster en Geografía, énfasis en Ordenamiento Territorial, EPG, Convenio UPTC-IGAC, Bogotá. Correo electrónico: damarychacon@hotmail.com
} 
Palabras clave: Zonificación ambiental, Desarrollo sostenible o sustentable, Reordenamiento territorial, Municipio Uribante.

\section{Abstract}

It shows part of the results obtained in the investigation developed by the author to opt for the Magister in Geography, with emphasis in Territorial Arrangement. The investigation responds to the latent necessity of defin ing a strategy directed to identify and to analyze the municipality Uribante's environmental problem, in the State of Táchira, Venezuela. It is sought to contribute with an environmental zoning's proposal applied to this area in particular, that allows to define and to propose alternatives to its development, coherent with the sustainability principles.

Key words: Environmental zoning, Sustainable development, Territorial reordering, Uribante municipality.

228 Damary Elizabeth Chacón Casique 


\section{Introducción}

El campo de acción de la ordenación del territorio es la interfase sociedadnaturaleza, y el enfoque más adecuado será aquel que logre integrar los elementos relevantes del sistema biofísico y del sistema socioeconómico, articulados a la estructura de base socio-territorial, de manera que, al tiempo de lograr el mejor uso del territorio, la localización adecuada de las actividades productivas y de los asentamientos humanos, así como el equipamiento territorial, se tenga en consideración la dinámica social y la estructura político institucional, articulada de manera armónica con la oferta de las condiciones y cualidades del medio biogeofísico (Méndez, 1996: 30).

Siendo así, el ordenamiento del territorio, cuyo origen responde a un intento de integrar la planificación socioeconómica con la física, debe contribuir a prolongar esa oferta de condiciones que brinda el medio biogeofísico, es decir, a la mejora y conservación del medio ambiente, o mejor, a lograr una equidad entre desarrollo económico y conservación de recursos. Aun así, en Venezuela este objeto no se ha logrado.

Se hace necesario, entonces, hallar soluciones concretas a una problemática común para nuestros países en vías de desarrollo, con el propósito de que se conozca la manera de actuar al ordenar un territorio, de modo que se integre el medio ambiente y lo afecte lo menos posible, para la preservación de especies y la elevación de la calidad ambiental, conjuntamente con la elevación de la calidad de vida del mismo ser humano.

La investigación realizada pretendió formular una propuesta de zonificación ambiental que pudiera servir de marco de referencia para el reordenamiento territorial que ha de llevarse a cabo en un futuro muy cercano en Venezuela, específicamente aplicable en ámbitos menores al nacional (a escala de regiones, subregiones o municipios). Propuesta que permita definir una estrategia orientada a la identificación de los principales problemas ambientales ya generados y las acciones pertinentes para poder mitigarlos y así evitar el deterioro de los recursos naturales existentes, para el logro de un verdadero desarrollo sostenible. Esta temática, que ha sido bastante abordada en los últimos tiempos, es, en efecto, un asunto de importancia capital en el Ordenamiento del Territorio, por cuanto la magnitud del deterioro ambiental de un espacio cualquiera constituye un elemento fundamental para su desarrollo y crecimiento.

La propuesta se formuló basada en el estudio de un espacio determinado del territorio venezolano: el municipio Uribante, estado Táchira, debido a que por sus características biogeofísicas constituye un espacio de riqueza incalculable para el estado Táchira y, por 
ende, para la región andina y para la nación; solo que las políticas actuales de planificación y de OT han subestimado su potencial.

Cabe destacar que el municipio Uribante es considerado como reservorio natural de agua, ya que por toda su geografía corren ríos de gran importancia que drenan hacia la cuenca del río Orinoco, como el Uribante, con un volumen que supera los 1.464 millones de $\mathrm{m}^{3}$, el Doradas y el Camburito, de similar caudal. El conjunto de este alto potencial hídrico permitió la creación del Complejo Hidroeléctrico Leonardo Ruiz Pineda, para su aprovechamiento eléctrico.

Con la investigación se quiso hacer un análisis de la situación ambiental que presenta el municipio Uribante, para detectar los principales problemas ambientales que han generado los usos indiscriminados del territorio debido a la explotación de sus recursos. Para ello fue necesario llevar a cabo la caracterización biogeofísica y socioeconómica del municipio, cuestión de vital importancia para el logro de la zonificación ambiental, pues esta información es su base y permite, al ser analizada, la interrelación entre las diferentes variables consideradas.

También fue necesario realizar un análisis de las distintas metodologías empleadas para la zonificación ambiental, como resultado de la síntesis del Plan de Ordenamiento Territorial, principalmente las usadas en Colom- bia, por cuanto en Venezuela no se le ha dado a este tema la importancia que requiere y muy poco se ha escrito al respecto; esto con el fin de proporcionar la base para la propuesta de zonificación ambiental que finalmente se realizó para el municipio objeto de estudio. Igualmente, como resultado final se presenta la zonificación del municipio. Cabe destacar que esta propuesta de zonificación se presentó en los términos más amplios posibles, dentro de lo que las limitaciones permitieron, por ser este un estudio que por su naturaleza requiere, primero, ser trabajado a una escala más detalla$\mathrm{da}$, y segundo, ser tratado por un grupo interdisciplinario de profesionales expertos en diversos temas.

\section{Zonificación ambiental para el ordenamiento territorial}

Para hablar de zonificación ambiental, antes debe aclararse el concepto de zonificación, que puede definirse como el proceso de división o parcelamiento, ya sea regular o irregular, en un área determinada, conducente a la definición de zonas individuales que poseen características propias y un grado relativamente alto de uniformidad interna en todos o en ciertos atributos esenciales para propósitos específicos.

Ahora bien, la zonificación ambiental del territorio se manifiesta como la expresión integral de los factores y procesos de formación del paisaje, $y$

230 Damary Elizabeth Chacón Casique 
del uso o aprovechamiento de los recursos naturales, tratando de minimizar su deterioro, para el logro de un desarrollo sostenible. Se define sobre la base de los resultados que se han diagnosticado, para configurar áreas homogéneas, con miras a una posterior reglamentación y asignación de usos del suelo, que constituye la base de la gestión ambiental. Todo ello para el logro de un posible manejo integral del paisaje y de los ecosistemas que lo conforman.

En el Plan de Manejo Ambiental, la Zonificación Ambiental se presenta, entonces, como el producto final, "donde se busca identificar las Unidades de Manejo Ambiental, cuya caracterización, espacialización y representación cartográfica constituyen el principal aporte para la planeación ambiental" (Torres, 1998: 19).

De modo que es necesario concebir que para realizar la zonificación ambiental se requiere, por un lado, conocer los procesos biogeofísicos y socioeconómicos que operan en el espacio estudiado, y, por otro, determinar el impacto que sobre la calidad de vida humana pueden llegar a tener ciertas acciones sobre la utilización de los recursos. Como dice Corpoguavio, "la zonificación [ambiental] tendrá en cuenta factores y elementos sociales, económicos, físicos y biológicos con manifestaciones visibles y cuantificables en lo posible, así como con repercusiones en la producción, bienestar de la población y protección del ambiente" (Colombia, 1988: 433).
En palabras de Méndez: "La zonificación ambiental viene a ser la expresión dinámica de la integración de elementos geoecológicos y socioeconómicos analizados, para configurar áreas socioambientales homogéneas sobre las cuales se pueden establecer reglamentaciones de uso" (1996: 109).

Ahora bien, diversas son las propuestas metodológicas con las que se ha intentado guiar la zonificación ambiental del territorio, cada una de ellas con diferentes énfasis y basadas en diversos principios o fundamentos. Para efectos de la investigación se seleccionaron algunas de estas propuestas, para ser analizadas y finalmente extraer de todas ellas los fundamentos que pudieran servir de base para la propuesta de zonificación ambiental.

\section{Antecedentes}

Como antecedentes de la investigación se tomaron las investigaciones realizadas por el Centro Interamericano de Desarrollo e Investigación Ambiental y Territorial (CIDIAT-Venezuela); los escasos estudios de orden geográfico e histórico realizados en el área de estudio, específicamente el estudio físico-histórico general realizado por el geógrafo José de la Cruz García y el estudio de la cuenca del río Uribante llevado a cabo por el Ministerio del Ambiente y los Recursos Naturales (MARN), y finalmente el Plan de Ordenación del Territorio del Distrito Uribante (hoy municipio). 
Debido a la escasa bibliografía existente en Venezuela en cuanto a metodologías concretas de zonificación ambiental para el ordenamiento territorial, también se tomaron como base para la presente investigación algunas de las metodologías propuestas en Colombia para zonificación ambiental, tales como: la propuesta hecha por el IGAC (Colombia, 1997a: 58-85), la utilizada para la Zonificación Ambiental de la Jurisdicción de Corpoguavio (Colombia, 1988: 51-76) y la utilizada para el municipio de El Cerrito por estudiantes y profesores del posgrado en Planeación urbano-regional de la UNAL sedes de Palmira y Medellín (Escobar, 2001: 517-537); así como otras propuestas de interés, como: la formulación del Plan Ambiental para la Cuenca Hidrográfica de la Laguna de Cucunubá (Villareal, 2000), la propuesta de planificación ambiental de la Corporación Autónoma Regional para la Defensa de la Meseta de Bucaramanga (CDMB) (Colombia, 1997b: 10-55), la propuesta de Zonificación Ecológica de la Región
Pacífica Colombiana (Colombia, 2000: 17-32) y la propuesta realizada por Corpochivor (Colombia, 1998b) en su asesoría para la formulación y puesta en marcha del OT de su jurisdicción.

\section{Localización y extensión del área de estudio}

El municipio Uribante se encuentra ubicado en la región de los Andes venezolanos, en la parte centro oriental del estado Táchira. Tiene una extensión territorial de $1.502 \mathrm{~km}^{2}$ y una población de 21.754 habitantes (según censo de 1990). La superficie del municipio corresponde al 13,4\% del territorio tachirense. Actualmente constituye el municipio más extenso de la entidad (ver mapa 1) ${ }^{* *}$. Políticamente se divide en una parroquia capital: Pregonero, y las parroquias: Potosí, capital Santa Marta; Juan Pablo Peñaloza, capital Laguna de García, y Cárdenas, capital La Fundación. Altitudinalmente se encuentra entre los 500 y 3.500 m.s.n.m.

\footnotetext{
** La escala de salida de los mapas originales es de 1:375.000, puede presentarse cierta distorsión al ser exportados del software Arc-view, en el cual se generaron, a un software de gráficos para efectos de la presente publicación.
}

232 Damary Elizabeth Chacón Casique 
Mapa 1

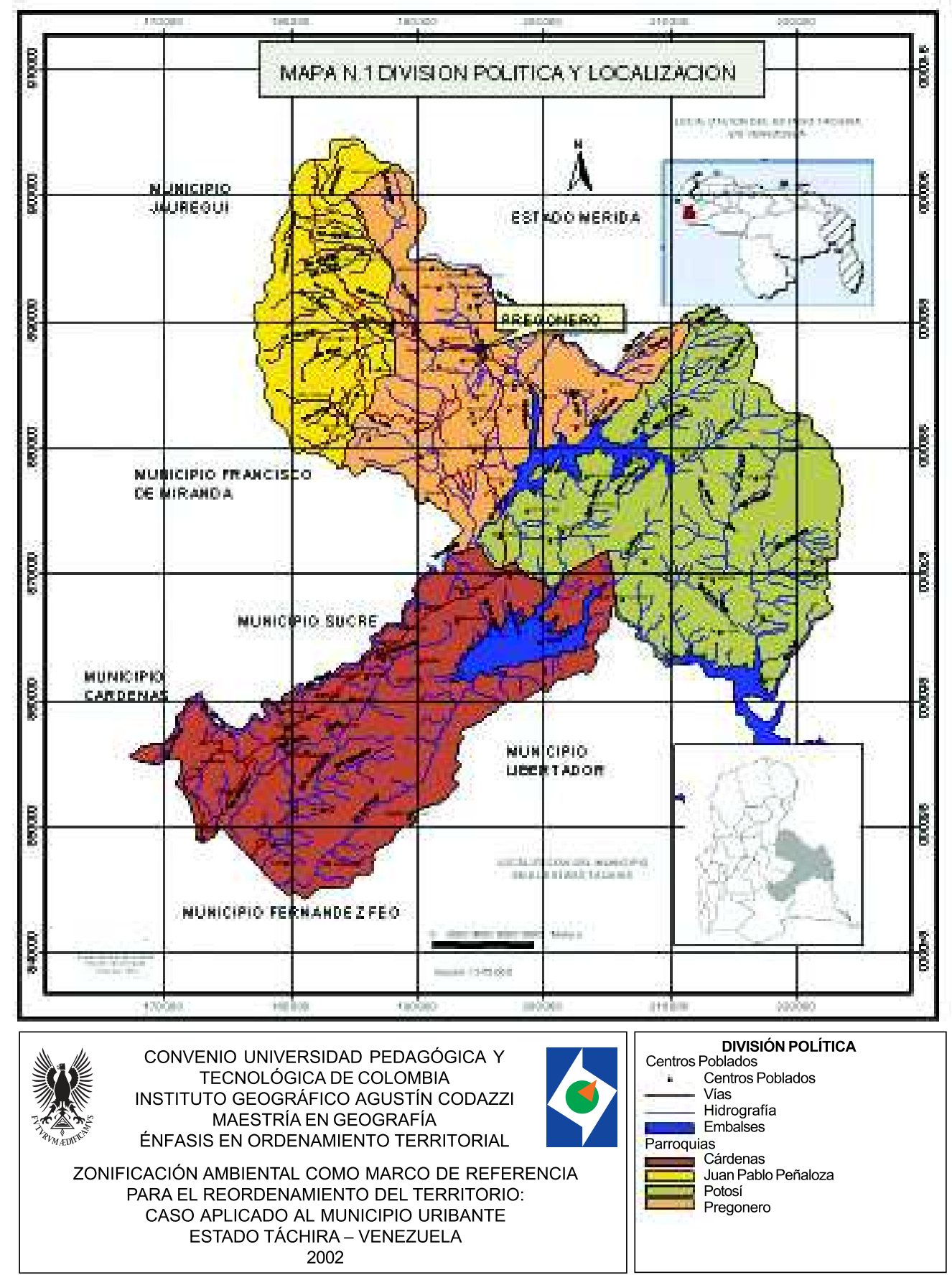

Perspectiva Geográfica Núm. 11, 2005 233 


\section{Identificación de variables}

Para el proceso de identificación de variables se seleccionaron aquellas que a juicio de la autora permiten caracterizar el medio ambiente del área estudiada en su integridad, de manera que se pueda identificar el deterioro ambiental del área de estudio y, con base en ello, realizar la respectiva zonificación ambiental. (La figura 1 muestra los aspectos que serán tomados en cuenta para la caracterización y estudio ambiental del municipio y las variables que serán consideradas para su respectivo análisis).

Cabe señalar que no se estudiaron otras variables de importancia para la investigación, pues se hace necesario, para el caso en particular, considerar la disponibilidad de información existente, el nivel de detalle del estudio, la disponibilidad de los recursos con los que se cuenta, tanto humanos como económicos y materiales, y las características particulares del área, entre otros.

Figura 1. Variables de la investigación.

A continuación se muestran de forma detallada las variables que se podrían utilizar para llevar a cabo una zonificación ambiental ${ }^{1}$, que no han de ser una camisa de fuerza y por ello se adaptarán a las consideraciones antes mencionadas:

1 Adaptación de la autora con base en los estudios de: Colombia. Ministerio del Medio Ambiente. Ordenamiento Ambiental Municipal. Bogotá: El Ministerio, $1998 \mathrm{~s} / \mathrm{n}$; Colombia. Minambiente - IGAC, op. cit., p. 30; Colombia. Minambiente - CDMB. Planificación Ambiental: Grado 09, op. cit., p. 49; Colombia. Corporación Autónoma Regional de Chivor. Op. cit., p. 7; Méndez, Elías. Op. cit., pp. 18-22.

234 Damary Elizabeth Chacón CasiQue 
- Factores bióticos. Como elementos primordiales se deben considerar:

- Vegetación y flora. En particular se debe estudiar las asociaciones vegetales, tipos de vegetación, estructura, fisonomía, composición florística, factores relacionados con la abundancia, densidad, dominancia, estabilidad, endemismos, estratificación, coberturas abarcadas y distribución de flora, las zonas protegidas relacionadas con la flora.

- Fauna. Se debe considerar es estudio de especies animales (acuáticas, aéreas y terrestres) en especial su abundancia, densidad, cobertura, dominancia, diversidad, representatividad, endemismos, distribución y zonas protegidas relacionadas con la fauna.

- Factores físicos o abióticos. Incluyen los siguientes elementos:

- Geomorfología. Unidades geomorfológicas, formas de relieve, y los procesos de morfogénesis, morfografía, morfometría, morfodinámica (mode-lamiento del terreno) e hidrodinámica.

- Geología. Litología, tipos, características y propiedades de las rocas, estructuras geológicas, tectónica, mineralogía, estratigrafía, geología estructural (lineamientos, fallas y contactos).

- Hidrología. Red de drenaje, caudales, cuerpos de agua, morfometría de cuencas, balance hídrico, áreas de recarga, zonas de acuíferos, niveles piezométricos, fuentes, características y calidad del agua.

- Edafología y suelos. Propiedades, características físico-químicas y clasificación de los suelos, aptitud de uso.

- Fisiografía. Pendientes, paisajes y topografía.

- Climatología. Temperatura, precipitación, vientos, evapotranspiración, brillo solar. atmósfera (calidad del aire), clasificación climática (Holdridge, Koeppen).

- Factores socioeconómicos. Incluye los siguientes elementos:

- Demografía. Crecimiento y distribución de la población, poblamiento y ocupación del territorio, composición de la población, estructura social y crecimiento, migración y densidad, fecundidad, mortalidad, estructura por sexo y edad, grado de urbanización o ruralización.

- Vivienda. Calidad, distribución y características de vivienda.

- Empleo. Índices, fuentes, distribución, estructura, procesos productivos, oferta de empleo, actividades económicas.

- Infraestructura y servicios. Servicios públicos básicos (acueducto, 
alcantarillado, energía), servicios sociales (educación, salud, recreación, protección social), otros servicios, redes de transporte y comunicaciones.

- Asentamientos. Redes de asentamientos, distribución, tipos, unidades político-administrativas, infraestructura habitacional.

- Usos del suelo. Usos urbanos, industriales, rurales; clases y tipos de uso de la tierra.

- Culturales. Patrimonio cultural: valor paisajístico y reservas indígenas. Actitudes, valores, tradiciones y costumbres; instituciones.

\section{Recolección de información}

\section{- Recolección de información prima-} ria. Para el análisis de las variables correspondientes a los aspectos físicos y bióticos, la información se verificó mediante la observación directa del área de estudio (trabajo de campo). Igualmente, se actualizó la cartográfica existente, sobre todo en lo que respecta al uso del suelo actual.

\section{- Recolección de información secun-} daria. Se obtuvo a través de algunos estudios realizados por investigadores independientes o por entidades estatales sobre la temática en cuestión, principalmente el Ministerio del Ambiente y de los Recursos Naturales (Venezuela).

Para la caracterización de los aspectos físico-bióticos se extrajo informa- ción de la cartografía existente, que, cabe mencionar, es bastante limitada, por cuanto a nivel municipal se conoció tan solo la generada por un estudio de suelos de la cuenca del río Uribante, por lo cual se trabajó con las cartas topográficas de la Dirección de Cartografía Nacional a escala 1:100.000, Núms. 5739, 5839 y 5840 que corresponden al área de estudio, complementado con la cartografía disponible a nivel estadal (estado Táchira), a escala 1:500.000.

Con ayuda del software ERDAS Imagine, se interpretó una imagen de satélite Landsant del Ministerio de Obras Públicas del Brasil, relativamente reciente (1998), que cubre parte del área estudiada, con el fin de hacer un diagnóstico general de las características relevantes del medio ambiente y establecer los posibles problemas de degradación del patrimonio natural del área de estudio, y también para actualizar la información suministrada por los estudios realizados en el municipio. Cabe destacar que aunque el nivel de detalle es muy general en este tipo de imágenes de satélite, se prefirió trabajar con esta imagen y no con fotografías aéreas, como fuera lo más indicado para este tipo de estudios, debido a que no existen fotografías aéreas recientes del área estudiada.

Los datos estadísticos correspondientes a los aspectos socioeconómicos se obtuvieron de la Oficina Central de Información (OCEI), con base en el último censo publicado (1990) y en las 
proyecciones realizadas por este mismo organismo. Cabe destacar que en Venezuela se llevó a cabo un último censo en el 2001, pero para la fecha en que se realizó la investigación sus resultados habían sido publicados.

Los datos suministrados por la OCEI fueron complementados con los del Censo Nacional Agrario (CENAGRI), que realizó la Dirección de Desarrollo Agropecuario, Industrial y Comercial (DAINCO) en 1995. Fueron tomados en consideración estos datos por cuanto son los más recientes y, además, porque la información se desagregó a nivel parroquial; pero ha de tomarse en cuenta que dichos datos se refieren a la población rural del municipio, específicamente a lo que en el censo denominaron "Unidades de Producción".

También se consideraron los datos suministrados por la Corporación Venezolana del Suroeste (CVS), antigua Corporación Regional del occidente del país, que hoy ya no existe, pero que suministró información de la región hasta 1998, específicamente en su División de Estadística e Información Regional.

Al obtener la información secundaria se procedió a jerarquizarla, ordenarla, sistematizarla y establecer las diferentes relaciones que puedan existir ente los elementos y variables, objeto de estudio.

Cabe destacar que la digitalización de todos los mapas se realizó utilizando el software AutoCad 2000; las opera- ciones de análisis espacial, a saber: superposición, clasificación, agrupación, entre otras, se realizaron con PC Arc-Info 3.4 y Arc-view 3.2. La interpretación de la imagen satelital se realizó mediante el software Erdas Imagine 8.5.

\section{Zonificación ambiental como resultado de síntesis}

El proyecto de zonificación que se presenta es el resultado final del proceso de análisis y síntesis de las variables consideradas para el estudio de caso; se constituye como la expresión final de la dinámica de los elementos analizados, tanto biogeofísicos, como socioeconómicos, con el fin de configurar unidades de manejo sobre las cuales se puedan establecer reglamentaciones de uso, que en últimas es la base de los procesos de gestión ambiental.

Se llevó a cabo un estudio general del municipio y, a partir de allí, se generó la cartografía temática con una escala de salida a 1:375.000. Se reconoce que este tipo de estudios exige un nivel de detalle mayor. Cabe destacar que, pese a que en un principio se proyectó realizar un estudio mucho más detallado, no se logró concretar, por cuanto no se contó con la información adecuada para tal fin.

Debido a las limitaciones de las resoluciones temporal y espacial de la información existente, el nivel de detalle con el cual se trabajó, si bien 
es cierto no es el idóneo para el desarrollo de un estudio de manejo ambiental como el que se propone, sí se consideró adecuado para constatar la aplicación real de la metodología propuesta, de modo que esta no se quedara en una simple disertación teórica.

Cabe señalar que, sea cual fuere el sitio de aplicación de la metodología para la zonificación ambiental, siempre se encontrarán algunas restricciones de diversa índole, de manera tal que se consideran un aporte de la investigadora los diversos mecanismos de adecuación a los que tuvo que llegar para poder trabajar con la información disponible, pero teniendo especial cuidado de no distorsionar los datos.

\section{Metodología integral para la zonificación ambiental}

Basado en algunas de las diversas metodologías ${ }^{2}$ existentes para zonificación ambiental, se procedió a generar un instrumento metodológico que permitió alcanzar la identificación de las unidades de manejo ambiental, correspondiente al resultado final de la aplicación de dicho instrumento.

Tomando como premisa el hecho de que la zonificación ambiental del municipio Uribante es tan solo un frag- mento dentro del proceso de ordenación territorial, que por su naturaleza, como se sabe, es bastante complejo e involucra otras dimensiones, la investigación realizada se vislumbra tan solo como un aporte al reordenamiento territorial que debe efectuarse en Venezuela, proponiendo una alternativa práctica para su desarrollo integral. En la figura 2 se muestra la secuencia metodológica que se siguió para identificar y analizar la problemática ambiental del municipio Uribante, teniendo como norte la búsqueda de alternativas de manejo y uso de los recursos naturales, que permitan su desarrollo sostenible.

El instrumento metodológico propuesto está basado en un marco de trabajo aproximado, entendiendo que, en la medida de los requerimientos, será posible incorporar elementos metodológicos, datos y conocimientos que permitan mejorarla y adaptarla para un área de aplicación particular, en ámbitos territoriales menores al nacional (a escala de regiones, subregiones o municipios).

La metodología consiste básicamente en una serie de procedimientos de recopilación, organización, análisis y síntesis de datos de diversa naturaleza multitemática, pues se plantea un enfoque integral del ambiente, donde se conjuguen aspectos tanto físicos, como sociales y económicos.

2 Fundamentalmente en la metodología usada por la Corporación del Guavio (Corpoguavio), que se adaptó tanto a las necesidades del municipio, como a los recursos e información primaria y secundaria existente.

238 Damary Elizabeth Chacón Casique 


\section{Primera fase. Preliminar}

Se partió de la premisa de que debían definirse los objetivos y alcances de la zonificación de acuerdo con las necesidades y particularidades del área de es- tudio, así como con las políticas y estrategias de los entes encargados tanto de la política ambiental como del ordenamiento territorial, que para el caso venezolano es el Ministerio del Ambiente y de los Recursos Naturales.

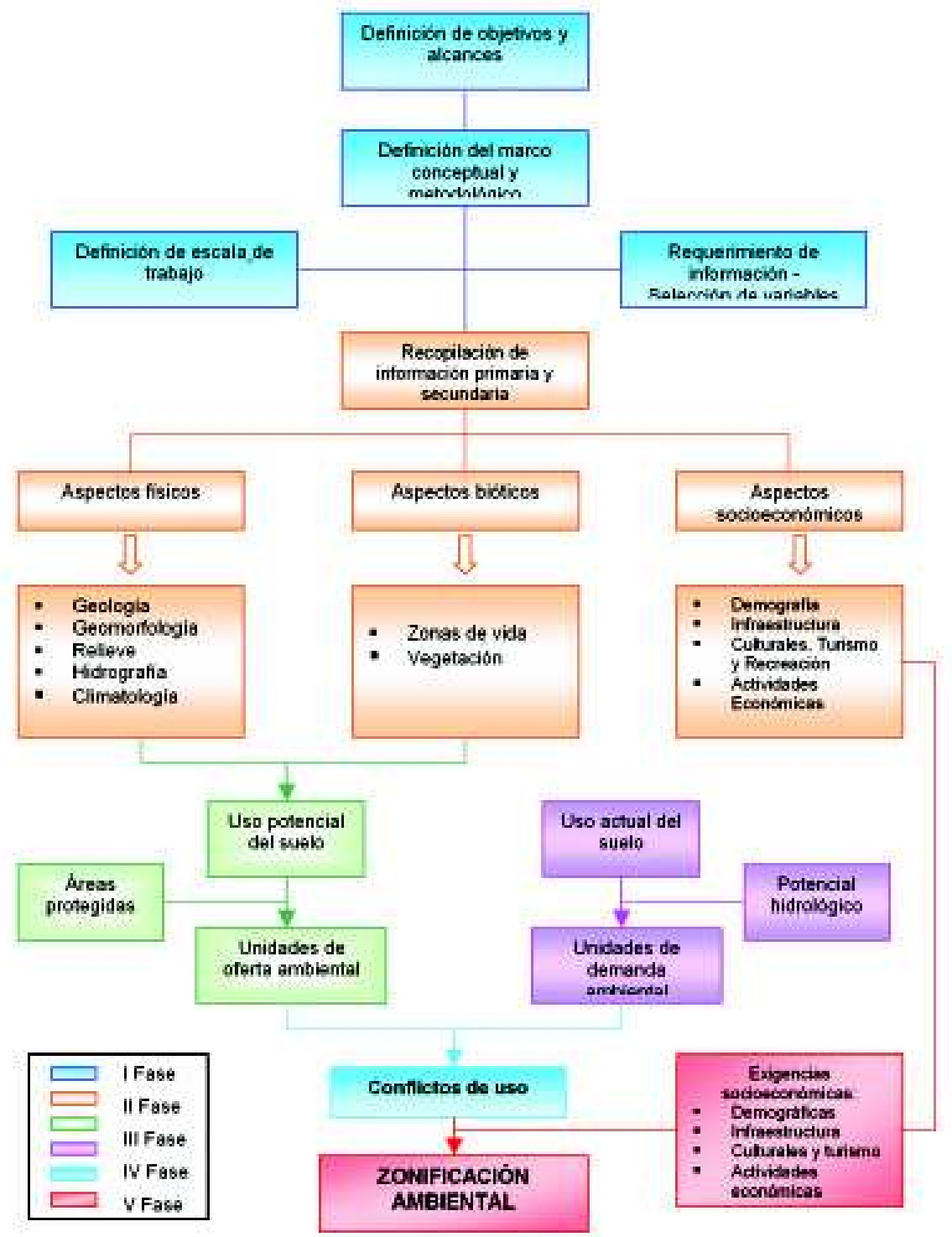

Figura 2. Secuencia metodológica para la zonificación ambiental. 
Luego de haberse definido los objetivos y alcances de la zonificación, se estableció un marco conceptual y un marco metodológico que pudiera servir de guía para su logro y, obviamente, para alcanzar los objetivos propuestos. Es en este momento en el que se estudiaron y definieron las aplicaciones metodológicas para estudios geográficos, ya establecidas, a ser utilizadas en el proceso de desarrollo de la zonificación, como es el caso de la metodología Holdridge, para la clasificación de las zonas de vida; la metodología Copen, para la identificación de las zonas climáticas, y la metodología definida por la División de Vegetación del Ministerio del Ambiente y de los Recursos Naturales (Venezuela, 1973: 187), para la determinación del uso del suelo actual.

Una vez adoptado el marco conceptual y metodológico, se procedió a identificar los requerimientos de información y la forma como esta se recolectaría. Esta actividad implica la selección de variables biofísicas y socioculturales (áreas temáticas), que permitan orientar el tipo de información requerida para el logro de los resultados. En este momento también se define el nivel de detalle idóneo para el estudio del área (escala de trabajo).

\section{Segunda fase. Diagnóstico}

Dentro de esta fase se recopiló la información primaria y secundaria necesaria para el estudio de las variables planteadas, correspondientes a los tres niveles de desagregación: aspectos físicos, aspectos bióticos y aspectos socioeconómicos.

Con base en la información primaria y secundaria, se procedió a realizar la caracterización de cada una de las variables (caracterización biogeofísica y socioeconómica), para determinar su comportamiento dentro del espacio geográfico. En este momento, la investigación se inclinó al procesamiento y análisis de la información recopilada de cada uno de los elementos temáticos.

Enseguida, y con base en lo anterior, cada una de las variables de orden físico y biótico fueron cartografiadas, así como algunas de las variables que integran el componente socioeconómico, generando mapas temáticos que permitieron el análisis interpretativo individual, necesario para luego ser interrelacionadas las variables entre sí.

Los mapas generados del componente físico fueron:

- mapa geológico

- mapa geomorfológico

- mapa de relieve

- mapa de zonas hipsométricas

- mapa de cuencas y microcuencas hidrográficas

- mapa de zonas climáticas

- mapa de zonas protegidas (ABRAES) 
Los mapas generados del componente biótico fueron:

- mapa de zonas de vida Holdridge

- mapa de vegetación

Los mapas generados del componente socioeconómico fueron:

- mapa de servicios públicos (contiene también la densidad de viviendas)

- mapa de servicios sociales (contiene también la densidad poblacional)

- mapa de actividades económicas (contiene también la densidad vial)

- mapa de turismo

- mapa de uso del suelo

\section{Tercera fase. Determinación de las unidades de oferta ambiental}

Con la combinación del componente físico y el biótico, y tomando en cuenta tanto las cualidades que ofrece el medio biogeofisico como los factores limitantes, se obtuvo el mapa de uso potencial del suelo.

Aunado a ello, considerando las áreas que por disposición del Ministerio del Ambiente se definen como Áreas Bajo Régimen de Administración Especial (ABRAES), se espacializaron estas áreas en un mapa, para superponerlo con el mapa de Uso Potencial del Suelo ya obtenido, y así se determinaron las unidades de oferta ambiental.

\section{Cuarta fase. Determinación de las unidades de demanda ambiental}

Por otro lado, para determinar las unidades de demanda ambiental se generó el mapa de uso del suelo actual. Para ello se utilizó como base el mapa de uso de suelo actual a escala 1:500.000 que reposa en la sede del MARN y, adicionalmente, se interpretó una imagen de satélite Landsat reciente, del Ministerio de Obras Públicas del Basil (1998), a escala 1:100.000, y se ensambló con el mapa antes mencionado.

Utilizando el mapa de potencial hidrológico generado por el estudio de la cuenca del río Uribante (estudio realizado por el MARN, y antes mencionado), se tomó en consideración la demanda y el uso del recurso hídrico. Este mapa se superpuso con el mapa de uso del suelo actual, lo que generó las unidades de demanda ambiental.

\section{Quinta fase. Determina- ción de unidades de conflicto}

Superponiendo las unidades de oferta con las unidades de demanda ambiental, se generó el mapa de zonas de conflicto (ver mapa 2). Tales conflictos se 
jerarquizaron como lo indica el cuadro 1. Cabe destacar que dicha jerarquización se realizó adaptando la propuesta realizada por Corpoguavio (Colombia, 1988: 463) para la identificación de áreas de conflicto.

Se adoptó esta propuesta de jerarquización de conflicto ambiental debido a que establece una clasificación que toma en consideración la ubicación del área propiamente dicha, es decir, toma en cuenta si esta se encuentra ubicada en zonas de aptitud ambiental o si, por el contrario, se ubica en zonas que pueden destinarse para la producción y desarrollo; cuestión que para el área objeto de este estudio es fundamental, debido a que gran parte de estas zonas que presentaron (o no) conflictos se encuentran sobre Zonas de Aptitud Ambiental, algunas, incluso, se encuentran protegidas (ABRAES).

\section{Cuadro 1. Tipos de conflictos ambientales}

\begin{tabular}{|c|c|c|c|c|c|}
\hline $\begin{array}{l}\text { Tipo de } \\
\text { conflicto }\end{array}$ & Ubicación & Categoría & Número & \multicolumn{2}{|c|}{ Símbolo } \\
\hline \multirow[t]{2}{*}{ Sin conflicto } & En zonas de aptitud ambiental & - & 1 & SC/AA & \\
\hline & $\begin{array}{l}\text { En zonas para la producción } \\
\text { y desarrollo }\end{array}$ & - & 2 & $\mathrm{SC} / \mathrm{P}$ & \\
\hline \multirow[t]{6}{*}{ En conflicto } & \multirow{3}{*}{$\begin{array}{l}\text { En zonas de aptitud } \\
\text { ambiental }\end{array}$} & Leve & 3 & C/AA-I & \\
\hline & & Moderado & 4 & C/AA-m & \\
\hline & & Severo & 5 & C/AA-s & \\
\hline & \multirow{3}{*}{$\begin{array}{l}\text { En zonas para la producción } \\
\text { y desarrollo }\end{array}$} & Leve & 6 & C/P-I & \\
\hline & & Moderado & 7 & C/P-m & \\
\hline & & Severo & 8 & $\mathrm{C} / \mathrm{P}-\mathrm{s}$ & \\
\hline
\end{tabular}

Para identificar las unidades de conflicto se utilizaron ciertos parámetros, que se explican a continuación:

\section{- Sin conflicto (SC)}

Se refiere a aquellas unidades en las que el hombre no ha intervenido para deteriorar el ambiente de una forma manifiesta, o bien, cuando se le ha dado un uso acorde con su potenciali- dad. A su vez, estas áreas se dividen en:

- Sin conflicto en zonas de aptitud ambiental (SC/AA). Se refiere a las zonas que por sus condiciones físiconaturales y por los recursos que involucran han sido declaradas bajo un uso protector, o bien, merecen una consideración especial de protección para su conservación o preservación.

242 Damary Elizabeth Chacón Casique 
Mapa 2

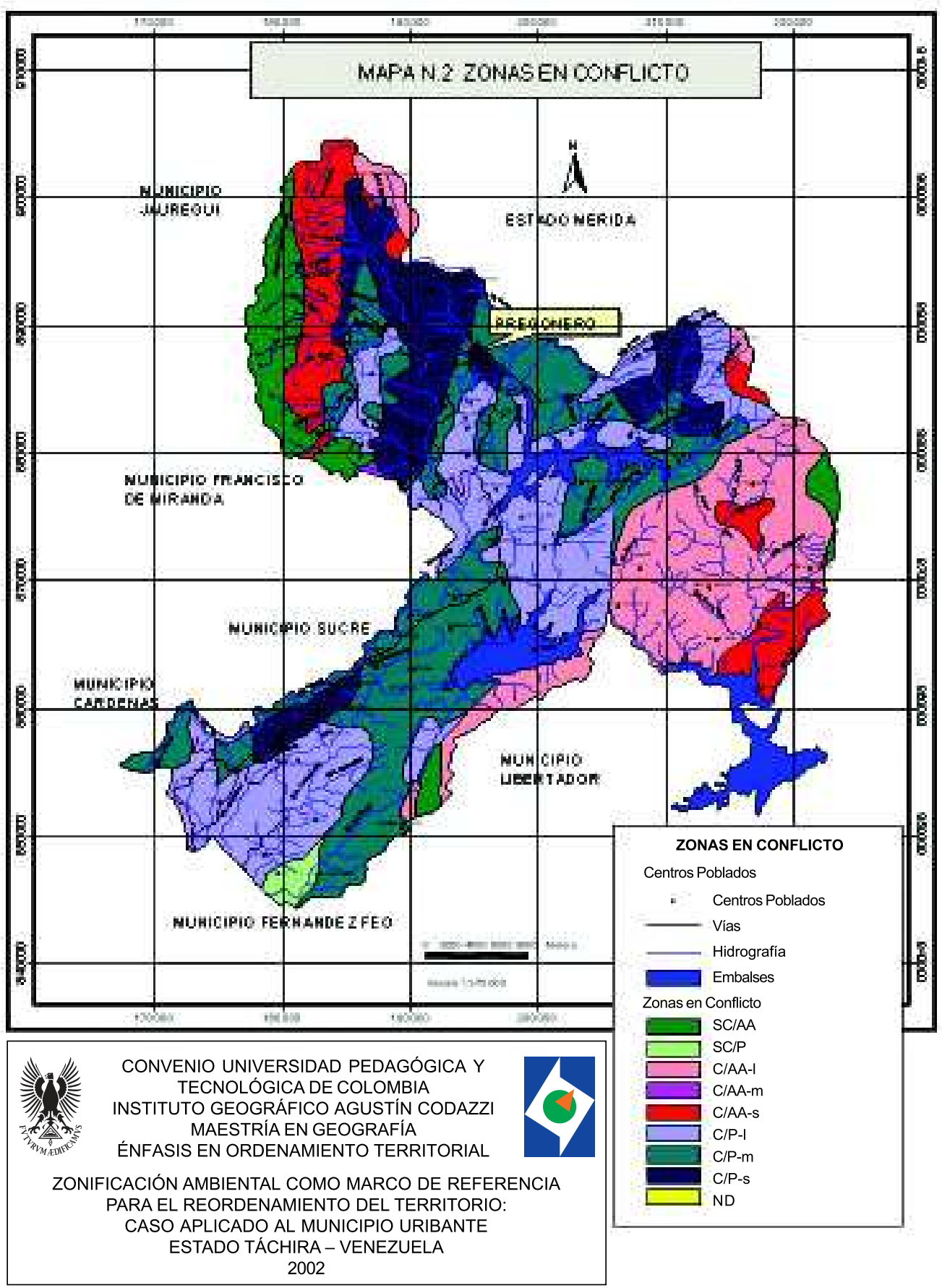


- Sin conflicto en zonas para la producción y desarrollo socioeconómico (SC/P). Corresponde a aquellas áreas cuyo uso corresponde con el uso potencial de ellas, es decir, los sistemas agrícolas, pecuarios, forestales, entre otros, se encuentran establecidos en las áreas que por sus condiciones biogeofísicas se definen para tal fin.

\section{- En conflicto (C)}

Se refiere a aquellas áreas en las que la posibilidad que brinda su potencial ambiental no se corresponde con el uso al que están siendo sometidas.

Al igual que las anteriores, se pueden ubicar en las zonas de aptitud ambiental o en las zonas para la producción y desarrollo socioeconómico. Las primeras se refieren a las áreas destinadas a protección, recuperación o preservación de los recursos naturales, cuyo uso no corresponde al que define su oferta, y las segundas, a aquéllas áreas destinadas a un fin productivo, o bien para su desarrollo, pero cuyo uso en la actualidad no es el que determina su potencial.

Los conflictos, según la intensidad y complejidad, se clasifican a su vez en tres categorías: leve, moderado y severo. Será leve cuando la oferta ambiental se encuentra afectada por el hombre en tanto que este subutiliza sus recursos; será moderado cuando el uso que el hombre le da a una zona determinada es incompatible con el uso potencial y genera un conflicto significativo, que requiere prácticas de recuperación para darles el uso adecuado, y finalmente, el conflicto se definirá severo cuando exista un contraste demasiado fuerte entre la oferta y la demanda ambiental, en cuyo caso requiere prácticas intensivas de recuperación a largo plazo para darle el uso que verdaderamente requiere.

Cuando el uso está dado por los asentamientos humanos o por los cuerpos de agua se infiere sin conflicto aparente. Para determinar los conflictos de uso en estas áreas se requiere de otro tipo de investigación o metodología.

El mapa obtenido de conflictos de uso determina, hasta ahora, la zonificación según la aptitud físico-biótica. Para obtener la aptitud biogeofísica y socioeconómica deberá confrontarse con las exigencias socioeconómicas de la población, es decir, con las variables: demografía, infraestructura básica, aspectos culturales, potencial turístico y actividades económicas.

Cabe señalar que antes de ello debe ser realizada una evaluación de amenazas naturales para el área estudiada, que se refiere al «estudio de la probabilidad de ocurrencia de un fenómeno natural potencialmente destructor dentro de un periodo determinado de tiempo, en un lugar geográfico específico» (Colombia, 1988: 464), clasificando las amenazas en alta, media y baja, en tanto que su efecto sobre el medio es 
determinante para establecer en qué medida está influyendo sobre este. Para efectos de la investigación efectuada, este estudio no se realizó, por cuanto corresponde a una investigación muy específica, en la que se requiere tanto de un experto en el tema como de cierta información preliminar. Sin embargo, cabe mencionar que al ser estudiadas las variables geología y geomorfología (aspectos físicos), se consideraron objeto de estudio para la zonificación ambiental las áreas de alta pendiente, los relieves dominantes y las zonas que por su composición geológica, son propensas a deslizamientos. No obstante, para la aplicación de la metodología, se sugiere realizar un estudio mucho más exhaustivo de amenazas naturales y generar el mapa correspondiente, don- de se delimitan las zonas de amenazas por actividad sísmica, por remoción en masa y por procesos de erosión, con el objeto final de establecer medidas de mitigación y prevención.

\section{Sexta fase. Determinación de las unidades de manejo}

Por otro lado, luego de haber sido analizadas las variables de orden socio-económico y de haberse cartografiado, en la medida de lo posible, se confrontaron con el mapa de conflictos de uso antes obtenido, lo que generó el mapa de zonificación ambiental final (mapa 3 ), en el que se determinaron las unidades de manejo (Colombia, 1988: 76), las mismas que se presentan en el cuadro 2 y se explican a continuación: 
Mapa 3

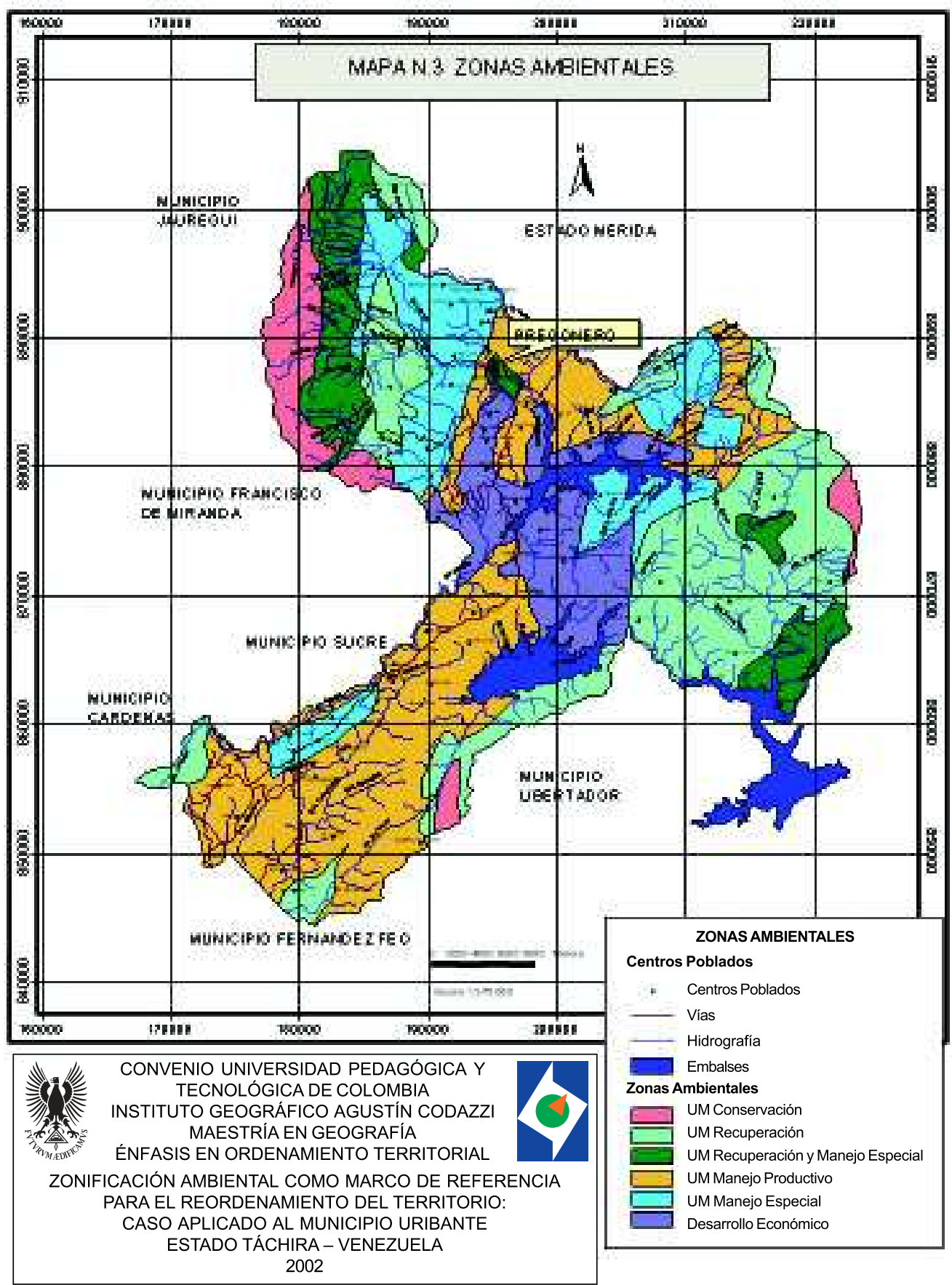

246 Damary Elizabeth Chacón Casique 
Cuadro 2. Unidades de manejo

\begin{tabular}{|c|c|c|c|}
\hline Ubicación & Unidades de manejo & & olo \\
\hline \multirow{3}{*}{$\begin{array}{l}\text { Zonas de manejo } \\
\text { ambiental }(A)\end{array}$} & Manejo para conservación & A-mc & \\
\hline & Manejo para recuperación & A-mr & \\
\hline & $\begin{array}{l}\text { Manejo con prácticas especiales } \\
\text { de conservación y recuperación }\end{array}$ & A-re & \\
\hline \multirow{3}{*}{$\begin{array}{l}\text { Zonas para la producción } \\
\text { y desarrollo } \\
\text { socioeconómico }(\mathrm{P})\end{array}$} & Manejo productivo & P-mp & \\
\hline & Manejo especial para cambio de uso & P-cu & \\
\hline & Desarrollo económico & P-de & \\
\hline
\end{tabular}

\section{- Zonas de manejo ambiental (A)}

Áreas que por sus características y su incalculable valor ecológico requieren un manejo dirigido a la preservación, recuperación, protección o prevención; en su mayoría se refieren a áreas que cumplen funciones ambientales de equilibrio o protección.

$\mathrm{Al}$ determinar estas áreas, lo recomendable es incluir las variables correspondientes a las áreas de riesgo y vulnerabilidad, así como las que en la actualidad presentan procesos de degradación de los recursos, que requieren el implemento de las prácticas de conservación. Dentro de estas áreas se determinaron tres unidades de manejo, a saber:

- Unidades de manejo para conservación. Áreas que se encuentran en equilibrio, sin intervención antrópica. Incluyen zonas de páramo, subpáramo y bosque alto andino (alturas superiores a 2.600 m.s.n.m.) que requieren ser protegidas por los entes competentes en materia ambiental, en la medida de lo posible, con la participación de la comunidad. Para el caso de Uribante se delimitaron estas áreas en el páramo El Batallón, al noroeste del municipio, en los límites con el municipio Jáuregui; en menor proporción al noreste del municipio, en los límites con el estado Mérida, y en un área menor al sur, en los límites con el municipio Libertador. Las dos primeras áreas se encuentran protegidas (ABRAES). Se recomienda su preservación.

- Unidades de manejo para recuperación. Áreas con dominio de clima frío en topografía superior al $75 \%$, que tienen cobertura de bosque primario o secundario intervenido. Para la delimitación de estas áreas se tomó en consideración, adicional a lo anterior, la variable 
Turismo y Recreación, en tanto que este es uno de los potenciales explotables por el municipio, dada la presencia de bosques que se pueden recuperar para el disfrute de la comunidad y de sus visitantes. Corresponden en su mayor parte a las áreas protegidas (ABRAES), en las que el uso dado al suelo no ha sido el más indicado. Se plantea la necesidad de mantener los bosques naturales en estos espacios, en tanto que su recuperación y conservación es de vital importancia para la conservación del recurso hídrico. Se recomienda conservación de bosques y reforestación natural, especialmente donde la pendiente es fuerte.

- Unidades de recuperación y manejo especial. Son aquellos espacios que han sido sometidos a procesos intensivos e inadecuados de intervención antrópica. Tal es el caso de las áreas que se encuentran bajo régimen de administración especial (ABRAES), especialmente las de páramo y nacientes de los ríos intervenidas por la acción antrópica. Esto implica que ese uso debe eliminarse, por lo que se requiere la aplicación de prácticas de recuperación. En el municipio estos espacios se localizan al este, en una pequeña proporción en los límites con el estado Mérida, y al noroeste, en las zonas bajas del páramo El Batalón, donde su vegetación natural de páramo sufre las consecuencias de ser desplazada, pese a ser esta un área protegida (ABRAE). Se recomienda desarrollar y propagar una agricultura orgánica para la zona de páramo y regenerar el bosque nativo. $\mathrm{El}$ MARN debe ejercer su función de organismo regulador.

\section{- Zonas para la producción y desarrollo socioeconómico (P)}

Aquellas zonas que por sus condiciones climáticas y edafológicas presentan capacidad y deben ser destinadas a las actividades vinculadas al desarrollo socioeconómico. Para precisar la localización de estas áreas se consideraron las exigencias socioeconómicas determinadas en el municipio, con base en la interpretación de su realidad sociocultural. Se determinaron en el municipio las siguientes áreas específicas:

- Unidades de manejo productivo. Tomando como premisa que la vocación del municipio es la actividad agrícola y pecuaria, se determinaron estas áreas, que por sus condiciones físicas podrían ser utilizadas para el desarrollo agropecuario. Estas unidades se distribuyen a lo largo de todo el municipio, pero predominan al sur, en la parroquia Cárdenas. Debe tenerse especial atención en no extender las fronteras que se proponen para este fin.

- Unidades de manejo especial (cambio de uso). Se refiere a aquellas áreas que están siendo sobreutilizadas, al extremo de que requieren un especial manejo de recuperación, 
considerando criterios que permitan su desarrollo sostenible. Se localizan al noreste del municipio, en la parroquia capital Pregonero, en las adyacencias al embalse La Honda donde el uso de fertilizantes y pesticidas en las actividades agrícolas está contaminando las cabeceras de los ríos-, y, en menores proporciones, distribuidas en el resto del municipio. Se recomienda el cambio progresivo de la agricultura química a una agricultura sostenible y una apertura comercial gradual de productos limpios. Utilización de sistemas silvo-pastoriles, especialmente en los valles de los ríos.

- Unidades de manejo para el desarrollo económico. Hace referencia a aquellas áreas propuestas para el desarrollo económico del municipio; para la delimitación de estas unidades se consideró la vocación turística de este; no obstante, debe atenderse con especial cuidado esta asignación, pues estas áreas no deberán ser utilizadas en forma desmesurada. Aunado a ello debe considerarse el hecho de que el municipio requiere una infraestructura acorde para el desarrollo de la alternativa que ofrece el turismo. Fueron localizadas en el área urbana de Pregonero, en las adyacencias del Embalse La Honda, por cuanto allí se encuentran las instalaciones La Trampa y La Tampita, del complejo Hidroeléctrico Leonardo Ruiz Pine$\mathrm{da}$, que aunque en la actualidad no presta sus servicios, fue considera- do a futuro. Recomendación: conservación de flora y fauna, y turismo ecológico.

Se logró obtener una visión general de la situación ambiental actual del municipio Uribante y generar propuestas o alternativas para su posible manejo. Esta es la alternativa que brinda la zonificación ambiental, vislumbrada como un fragmento dentro del proceso de ordenamiento territorial.

\section{Conclusiones}

La zonificación ambiental se presenta como un instrumento metodológico que puede servir de aporte en la elaboración de los Planes de Reordenamiento Territorial, pues la zonificación proporciona la localización geográfica y el inventario de áreas que presentan características físicas, bióticas, socioeconómicas y culturales propias, que las diferencian de otras, lo cual permite su uso sostenido.

La caracterización biogeofísica y socioeconómica del municipio Uribante permitió interpretar, analizar, sintetizar, evaluar y explicar la realidad ambiental del municipio, lo que hizo posible, en la medida del cumplimiento de la metodología propuesta para la zonificación ambiental, configurar unidades de manejo sobre las cuales se pudieran establecer reglamentaciones de uso. Así, este instrumento metodológico podría ser considerado como un instrumento de apoyo para el proceso de reordenación territorial 
que en un futuro muy próximo ha de realizarse en Venezuela.

El instrumento metodológico propuesto se basó en un marco de trabajo aproximado, entendiendo que, en la medida de los requerimientos, es posible incorporar elementos metodológicos, datos y conocimientos que permitan mejorarlo y adaptarlo para un área de aplicación particular, en ámbitos territoriales menores al nacional (a escala de regiones, subregiones o municipios).

El municipio Uribante presenta problemas de diversa índole, que podrían mitigarse con el manejo, uso adecuado y gestión coherente de los recursos con que cuenta. Estos problemas son, entre otros: la carencia de políticas y planes de desarrollo agropecuario acordes con la potencialidad de los recursos suelo y agua; el estancamiento de la dinámica de crecimiento y desarrollo agrícola, se presume que por falta de planes crediticios y asistencia técnica para el medio rural; la pérdida de la capacidad productiva de los suelos por las inadecuadas prácticas agrícolas; la tala, quema y destrucción de la vegetación en nacientes y cursos de agua, que están poniendo en peligro de desaparecer a algunas quebradas y ríos; los insuficientes programas de conservación para el logro del desarrollo sostenible en el municipio; pese a que gran extensión del municipio se encuentra bajo régimen de administra- ción especial, no son suficientes los esfuerzos por parte del MARN para cumplir estas pautas; la masa boscosa del municipio es escasa y está siendo destruida por la tala y la quema; el área rural del municipio presenta deficiencia en su infraestructura básica.

Aunque se cuenta con un Plan de Ordenamiento del Territorio para el municipio, se presentan conflictos de uso, porque no se toman en cuenta las propuestas que en él se plantearon; el MARN, organismo encargado de su seguimiento y control, no ha cumplido con su función. Por otra parte, el Plan de Ordenamiento Territorial elaborado en 1996 solo consideró variables físico-bióticas para la determinación de áreas de uso. Dichos planes requieren ser retomados. En Venezuela se habla de los Planes de Reordenamiento Territorial, como futuro próximo dentro de las políticas de Estado.

El medio ambiente deberá ser contemplado como un sistema en el que se conjugan múltiples variables de orden biogeofísico y socioculturales, que permiten su análisis integral. El trabajo de investigación proporciona lineamientos generales en cuanto a la situación ambiental del municipio, necesarios para abordar políticas de reordenación de su territorio (en su dimensión ambiental), tan necesarias para su crecimiento y desarrollo sostenible. 


\section{Bibliografía}

ÁNGeL, E. et al. (1997): Gestión ambiental en proyectos de desarrollo. Santafé de Bogotá: FEN. 233 p.

Colombia, Corporación Autónoma Regional del Guavio (1988): Zonificación Ambiental Jurisdicción CORPOGUAVIO. s/1: La Corporación. 602 p.

, Corporación Autónoma Regional de Chivor (1998): Asesoría, soporte y apoyo para la formulación y puesta en marcha de los esquemas de ordenamiento territorial en la jurisdicción de CORPOCHIVOR. Garagoa: CORPOCHIVOR. 40 p.

, Instituto Geográfico Agustín Codazzi (1997a): Guía Metodológica para la formulación del Plan de Ordenamiento Territorial. Bogotá: IGAC. 186 p.

, Corporación Autónoma Regional para la Defensa de la Meseta de Bucaramanga -CDMB- (1997b): Planificación Ambiental: Grado 09. Bucaramanga: Minambiente. pp. 10-55.

, Ministerio del Ambiente, Instituto Geográfico Agustín Codazzi (2000): Zonificación Ecológica de la Región Pacífica Colombiana. Bogotá: Minambiente - IGAC. pp. 17-32.

Escobar, Carlos et al. (2001): "Zonificación Ambiental del municipio de El Cerrito con miras a un ordenamiento territorial". En: Espacio y Territorios: Razón, pasión e imaginarios. Bogotá: UNAL/RET, pp. 535-547.

Espinal, Sigifredo y E. Montenegro (1977): Zonas de vida o formaciones vegetales de Colombia: Memoria Explicativa sobre el Mapa Ecológico. Instituto Geográfico Agustín Codazzi, Departamento Agrológico. Bogotá: IGAC.

EstabA, Rosa (1999a): "La descentralización y la ordenación del territorio en Venezuela: estrategias hacia la modernidad". Scripta Nova Núm. 54 [diciembre], Revista Electrónica de Geografía y Ciencias Sociales. Universidad de Barcelona. España.

(1999b): Gestión ambiental en Venezuela: 1976-1995. Mimeo, en elaboración. Seis capítulos.

García M., José (1990): Uribante: geografía e historia. San Cristóbal: el Autor. 90 p.

GómEz O., Domingo (1994): Ordenación del Territorio. Una aproximación desde el medio físico. Madrid: Agrícola Española. 128 p.

Hunt, David et al. (1996): Sistemas de gestión medioambiental, principios y práctica. Madrid: McGraw-Hill.

MaLavé, José [comp.] (1988): La gestión ambiental: ¿Impulso o freno al desarrollo? Caracas: IESA, pp. 17-38.

MÉNDEZ V., Elías (1996): Planificación ambiental y desarrollo sostenible. Mérida: CIDIAT. $144 \mathrm{p}$.

Perspectiva Geográfica Núm. 1 1, $2005 \quad 251$ 
RodRíGuez, J. C. (1995): "Planificación urbana y descentralización político-administrativa en Venezuela (1984-1994)". Revista Latinoamericana de Estudios Urbano-Regionales $\mathrm{xxI}(64)$ : 37-60. Venezuela: EURE. .

ToRres M., Myriam (1998): Zonificación ambiental de una cuenca hidrográfica. Bogotá: Convenio Sena-Ministerio del Ambiente. 105 p.

Tratado de Cooperación Amazónica (1998): Zonificación ecológica-económica: una propuesta metodológica para la Amazonía. Caracas: El Tratado. pp. 5-103.

Venezuela, Comisión Nacional de Ordenación del Territorio (1998): PNOT Un Acuerdo a 20 años. Caracas: PDVSA. Año 1, Núm. 1. 112 p.

, Congreso de la República (1983): Ley orgánica para la ordenación del territorio. Caracas: El Congreso. 48 p.

, Dirección de Desarrollo Agropecuario, Industrial y Comercial -DAINCO(1995): I Censo Agrario del Estado Táchira '95. San Cristóbal: Gobernación del Estado Táchira. $71 \mathrm{p}$.

, Estado Táchira. Comisión Regional de Ordenación del Territorio - División de Planificación y Ordenación del Ambiente (1987): Plan de ordenación del territorio Estado Táchira. San Cristóbal: La Comisión. Vol. I. 220 p.

, Estado Táchira. Comisión Regional de Ordenación del Territorio - División de Planificación y Ordenación del Ambiente (1987): Plan de ordenación del territorio Distrital: Distrito Uribante. San Cristóbal: La Comisión. Vol. IV, pp. 804844.

, Ministerio del Ambiente y de los Recursos Naturales Renovables (1986): Atlas del Estado Táchira. San Cristóbal: Dirección de Cartografía Nacional, p. 01 - VI-06.

, Ministerio del Ambiente y de los Recursos Naturales Renovables (2000): Estudio de suelos de la cuenca del río Uribante, Estado Táchira. Informe Técnico. San Cristóbal: MARN, pp. 2-25.

, Ministerio del Ambiente y de los Recursos Naturales Renovables (1973): Metodología para la determinación del uso de suelo actual en la región Los Andes. Caracas: División de Vegetación MARN. 187 p.

, Oficina Central de Estadística e Información. Publicaciones Electrónicas (1998): Municipio Uribante. San Cristóbal: OCEI, s/p.

Villarreal, Jaime (2000): Cucunubá: Modelo para un desarrollo sostenible. Bogotá: Universidad de Bogotá Jorge Tadeo Lozano. 275 p.

Fecha de recepción: marzo de 2003

Fecha de aprobación: agosto de 2003

252 Damary Elizabeth Chacón CasiQue 